\title{
Performance Improvement of Multi-User Multiple-Input Multiple-Output Protocol for WLAN
}

\author{
Maha Bakalla, Mznah Al-Rodhaan, Yuan Tian \\ Department of Computer Science, College of Computer and Information Sciences King Saud University, Riyadh, KSA \\ Email:mahabakalla@hotmail.com,rodhaan@ksu.edu.sa,ytian@ksu.edu.sa
}

How to cite this paper: Bakalla, M., Al-Rodhaan, M. and Tian, Y. (2017) Performance Improvement of Multi-User Multiple-Input Multiple-Output Protocol for WLAN. Communications and Network, 9 , 124-141.

https://doi.org/10.4236/cn.2017.92008

Received: February 20, 2017

Accepted: May 24, 2017

Published: May 27, 2017

Copyright ( 92017 by authors and Scientific Research Publishing Inc. This work is licensed under the Creative Commons Attribution International License (CC BY 4.0).

http://creativecommons.org/licenses/by/4.0/

\begin{abstract}
The increase in the number of devices with a massive revolution in mobile technology leads to increase the capacity of the wireless communications networks. Multi-user Multiple-Input Multiple-Output is an advanced procedure of Multiple-Input Multiple-Output, which improves the performance of Wireless Local Area Networks. Moreover, Multi-user Multiple-Input Multiple-Output leads the Wireless Local Area Networks toward covering more areas. Due to the growth of the number of clients and requirements, researchers try to improve the performance of the Medium Access Control protocol of Multi-user Multiple-Input Multiple-Output technology to serve the user better, by supporting different data sizes, and reducing the waiting time to be able to transmit data quickly. In this paper, we propose a Clustering Multi-user Multiple-Input Multiple-Output protocol, which is an improved Medium Access Control protocol for Multi-user Multiple-Input Multiple-Output based on MIMOMate clustering technique and Padovan Backoff Algorithm. Utilizing MIMOMMate focuses on the signal power which only serves the user in that cluster, minimizes the energy consumption and increases the capacity. The implementation of Clustering Multi-user Multiple-Input Multiple-Output performs on the Network Simulator (NS2.34) platform. The results show that Clustering Multi-user Multiple-Input Multiple-Output protocol improves the throughput by $89.8 \%$, and reduces the latency of wireless communication by $43.9 \%$ in scenarios with contention. As a result, the overall performances of the network are improved.
\end{abstract}

\section{Keywords}

Clustering Multi-User Multiple-Input Multiple-Output, Multi-user Multiple-Input Multiple-Output, MIMOMate, Padovan Backoff Algorithm, Wireless Local Arewa Network 


\section{Introduction}

Wireless Local Area Network (WLAN) becomes an important technique in our life in recent years. However, the applications that used this technology require a lot of bandwidth. Telecommunications manufacturing plays an important role in wireless communication, which leads to the widespread acceptance of cell phones and wireless computer networking. Moreover, the number of users who used the network increases dramatically. Therefore, the capacity of current wireless communications networks has also increased. As a result, services require high data rate, great capacity, high quality, growth throughput and spectral efficiency. Therefore, various technologies are proposed to improve the wireless networks to meet the demands of users [1].

The latest technology is Multi-user Multiple-Input Multiple-Output (MUMIMO), which produces by IEEE 802.11ac to improve the efficiency of WLAN. Modifying the Medium Access Control (MAC) protocol in IEEE 802.11ac is the new research area. One of the existing technologies that modified the MAC protocol to work on the MU-MIMO introduces the MIMOMate technique. It forms a cluster for users in the Access Point (AP) in a way to increase the throughput because the throughput of a user highly relies on who transmits simultaneously with it. This technique tries to maximize the gain of MU-MIMO, and to reduce the overhead on the network by allowing the leader of each cluster to content instead of all users using standard 802.11 carrier sense multiple access with the collision avoidance (CSMA/CA) [2]. This work does not explain and clarify the details of communication such as: how the contention between leaders will be, how the members of that leader know that they have to send now, and how different antennas and AP will be communicated together. Moreover, it does not determine when the new contention will start if the current transmission end. In addition, it uses the original backoff algorithm in its CSMA/CA protocol, which is Binary Exponential Backoff (BEB). Our proposed algorithm Clustering Multi-user Multiple-Input Multiple-Output (CMU-MIMO) is designed to improve the communicational aspects in MU-MIMO by utilizing MIMOMates technique and trying to enhance the communication between different antennas to improve the throughput and reduce delay. Firstly, improving the MU-MIMO MAC protocol by applying an efficient Padovan Backoff Algorithm (PBA) in the IEEE 802.11. This algorithm will improve the network performance by enabling a huge reduction of collisions when many users try to entrance the shared wireless medium simultaneously. Also, after making clusters by using MIMOMate, we explain how the contention between leaders will be, and how the members will communicate with the antennas. Moreover, we will discuss about how these antennas will communicate together to service these users. Finally, determining the start of another new transmission. As a result, the MU-MIMO will cover more areas in WLAN, in the term of providing high throughput due parallel data channeling, increasing the capacity by implementing MU-MIMO with MIMOMate in wireless networks, focusing the signal power to serve only the user in that cluster, minimizing the energy consumption and reducing latency of wire- 
less communication.

The remainder of this paper is organized as follows: Section 2 discusses the background of the main concepts of the paper. Section 3 presents the literature review related to the MU-MIMO MAC protocols. The design of CMU-MIMO protocol that improves the IEEE 802.11n protocol is explained in Section 4. In Section 5, the experiment results are discussed. Finally, the conclusion and future work are presented in Section 6.

\section{Background}

Multiple-Input Multiple-Output (MIMO) communication has experienced a conversion from a theoretical concept to a real-world technique in last few years for improving the performance of wireless networks. Studying MAC mechanism for MU-MIMO in WLAN is the main point in this paper. Therefore, this section gives the foundation that helps to understand the basic principles.

\subsection{IEEE 802.11 Wireless Network}

A combination of the physical layer and MAC are the main principles of IEEE 802.11 WLAN. The MAC layer is the lower sublayer of the Open Systems Interconnection model; it allows many stations to share a single wireless channel. Meanwhile, the IEEE 802.11 MAC technique only allows one communication at the same time. Due to this limitation, there is no benefit of using MU-MIMO, therefore, MU-MIMO has tried to adapt their MAC protocol and their methods to control the simultaneous transmission between stations [3].

\subsection{Overview of MIMO}

MIMO is an improved smart antenna that used in wireless communication. MIMO is a complex technology, although it is widely used for wireless communication as it offers high data rate and throughput without additional bandwidth or spectral frequency. There are two operational modes in MIMO systems: the single-user and multi-user MIMO technologies, respectively.

In the single-user MIMO system, there are only one receiver and one sender, each of them has multiple antennas, which is a point-to-point communication. In each round, only one station can use the channel to send and receive multiple data so, it does not support sending data to multiple receivers at the same time. Whereas MU-MIMO is a point-to-multi-point network communication, in this technique, there are many receivers sending/receiving data to AP, and each of them has many antennas [3].

\subsection{Quality of Services Concept}

The communication through network forms as the basis of any successful communication such as in organizations and institutions. These networks transmit many data that has to reach to users with high resolution and without any delay or error. The set of performances that manage these networks are called quality 
of services. One of these performance matrices is throughput, which measures the success amount of transmitted data.

In Distributed Coordination Function scenario, each station has its Contention Window (CW) that starts from zero and increases exponentially with each unsuccessful transmitting by using BEB. Also, Distributed Coordination Function delegates stations to keep listening to the network channel. If it is idle for Distributed coordination function Inter Fame Space (DIFS), every station starts reducing a backoff timer that is selected from its CW. The station has fewer backoff successes to access the channel and sends its data. The collision happens if many stations select the same random backoff, In this case, the senders increase their CW and then try to access the channel again [3].

\section{Literature Review}

Recently, MIMO and its versions are a popular technology in wireless communications and there are extensive studies go through it. This chapter provides a comprehensive literature review on the availability of multi-user medium access control and backoff algorithms in WLAN, which has a strong influence on the development of new MAC protocol.

\subsection{MIMO in Wireless Area Network}

An asynchronous MIMO Multiple Packet Reception method is proposed in [4]. The MAC algorithm in this method operates to expand the reception duration on the receiving side of the standard IEEE request-to-send and clear-to-send (RTS/CTS) scheme to enable for obtaining a larger number of received requestto-send frames. The Network Simulator (NS-2) is used to compare the proposed MAC protocol with the IEEE 802.11 standard. The simulation results show that IEEE 802.11 achieves better outcomes for greater values of backoff. In contrast, Multiple Packet Reception-aware MAC achieves good results in a small value of the minimum CW size, because most collision transforms to double successful transmissions for the Multiple Packet Reception-aware MAC. As a result, window sizes are kept at their smallest values.

A whole distributed random access protocol for designing and implementing of 802.11n+ for MIMO wireless networks is presented in [5]. The objective of this design is to enable as many simultaneous transmissions as possible by the MIMO transmitter with a large number of antennas. In this design, the stations with extra antennas than the contention winner continue to sense and contend for the channel. Analysis scenarios are shown where the sender and receiver have the same or a different number of antennas. The result compares the new design against the existing $802.11 \mathrm{n}$ design in the term of throughput and shows that the throughput increases when the number of antennas increased.

\subsection{MU-MIMO MAC Protocol}

It is important to design precoding schemes in MU-MIMO system to support co-channel interference in the downlink. Therefore, researchers in [6] designed a 
precoder by maximizing the signal-to-leakage-and-noise ratio for all clients simultaneously. They compared their method with the zero-forcing precoding technique that forces a constraint on the relation between the numbers of transmitting and receiving antennas. The proposed method outperforms the zero-forcing precoding even when the dimension requirement for zero-forcing solutions is satisfied.

Analyzed and compared two schemes which are single-user MIMO and MUMIMO with random station distribution scenarios in [7]. In single-user MIMO scheme, there are many symbols transmitted from one station, while in MUMIMO scheme from many stations as in Multi-Packet Reception in uplink WLAN. Moreover, analyzing and comparing the maximum throughput achievement of both the single user and MU-MIMO schemes in uplink WLANs mode. The result shows that the MU-MIMO scheme is a good elect for enhancing the effectiveness of the network because it produces high throughput performance for few data load sizes and a huge amount of stations competing, however, the single-user MIMO scheme shows that is uselessness for small-sized packets.

\subsection{MAC Protocol in MU-MIMO Uplink}

A new cluster-based carrier sense multiple access with the collision avoidance scheme is introduced in [8]. This scheme is simple and could be utilized to a distinct infrastructure or ad hoc network. In this protocol, stations and AP are allocated different clusters and stations within the same cluster can send concurrently. Each cluster shares a single backoff, by this the cluster is seen as one node in the wireless network. The contention is done between stations in a different cluster. The result of this proposed method shows that it decreases the collision chance in a network and provides throughput enhancement compared to the other system based on 802.11 .

To maximize the gain of MU-MIMO and provides fairness for clients, a new user matching MAC protocol is present in [2] which is MIMOMate, a leader-contention-based MU-MIMO MAC protocol. In this protocol, the clients are groups in MIMO-Mates, each MIMO-Mate can send at the same time with only one contending process. The contention is done between the leaders of each group by using a traditional CSMA/CA. The MAC overhead is reduced because it needs a single contention for concurrent transmission. The implementation results and the theoretical analysis proved that this matching protocol could achieve greater throughput compared to the conventional contention scheme using two antenna AP scenarios.

\subsection{Backoff Algorithms}

In order to enhance the effectiveness of 802.11 MAC protocol in Distributed Coordination Function mode, a new Backoff scheme called PBA is proposed in [9]. This algorithm reduces the waiting time and delay of nodes in the network. It allows CW to increase as Padovan sequences instead of exponentially as BEB. The simulation results shows that the PBA allows more effective network re- 
sources utilization and beats the standard BEB scheme in diverse scenarios. In addition, it realizes greater throughput compared to $\mathrm{BEB}$, particularly in a dense network.

After analyzing the behavior of BEB, an Improved Binary Exponential Backoff algorithm is proposed in [10] to enhance the fairness of medium access which is missing in BEB. This improvement is made by modifying the way of growing or shrinking the CW based on a number of frames that transmit successfully. The simulation results show that the Improved Binary Exponential Backoff enhances the fairness and throughput capacity compared to BEB in both the static and dynamic environments.

A new Enhanced Binary Exponential Backoff algorithm is introduced in [11], to enhance the fairness of channel access in the network. The improvement in Enhanced Binary Exponential Backoff is made by offering some association between Backoff Time Threshold and CW size. To reduce collisions, if there are several users consuming the channel, it needs a small value of Backoff Time Threshold comparative to present CW size, and via verse. The simulation is performed by using NS-2.34, and the results show that Enhanced Binary Exponential Backoff is better than BEB and Improved Binary Exponential Backoff algorithms [10] regarding fairness and throughput.

\section{Clustering MU-MIMO Protocol}

The MAC layer provides channel access control mechanisms to allow several clients to incorporate a shared medium and communicate within a multiple access network. As a result, there are many issues come up with this concept, such as packet collision, delay, and fairness between users. Therefore, the need for designing MAC protocol to deal with these issues has been an active area of research. In this section, the main idea and the detailed design of CMU-MIMO MAC protocol are presented. CMU-MIMO protocol applies Padovan backoff protocol. In addition, it improves MU-MIMO protocol by using the MIMOMate clustering technique.

\subsection{Padovan Backoff Algorithm}

Wireless networks indicate using radio frequency signals to exchange information and communication among wireless devices. IEEE 802.11 protocol is a famous and extensively used as core MAC protocol in WLAN. With advancing years, the numbers of users that use the wireless network are increasing. As a result, when users try to enter the shared wireless channel simultaneously, it leads to increase the collision problem that will reduce the network performance in the distributed MAC protocol under Distributed Coordination Function mode. To avert such collisions or at least decrease it, the well-known algorithm is used which is BEB [12].

$\mathrm{BEB}$ algorithm tries to increase the $\mathrm{CW}$ of users exponentially after every ineffective transmission. Consequently, it may cause an under-utilization of the medium bandwidth, which leads to having needless idle time in the medium. As 
a result, it leads to increase the delay, lack fairness, low throughput and reduce the overall network performance, especially in the dense network. In this network there are a lot of users that want to occupy the shared channel. Sequential, collisions have a high probability to happen in such type of network. Accordingly, increasing the CW exponentially is not the best solution to deal with the packet loss problem. Since the time selected from the CW might be greater than the required waiting time to guarantee collision-free channel access [9].

In this paper, we use $\mathrm{PBA}$, to overcome the above problems that faced $\mathrm{BEB}$ algorithm. PBA will allow a significant decreasing of the number of collisions and guarantee better controlling of the $\mathrm{CW}$ size based on the Padovan sequence number. The main goal of using PBA is to enhance the effectiveness of our IEEE 802.11 MAC protocol and reduce delay when working in Distributed Coordination Function mode and dense network.

\subsection{CMU-MIMO Protocol}

Nowadays, the numbers of clients use the wireless network to communicate concurrently are increasing. Consequently, the needing to explore a new technology to deal with them is increasing. MU-MIMO technology is an exciting development standard that evolved to enable multiuser sending and to receive at the same time. To achieve the benefits of having MU-MIMO technology, we have to consider a good matching clustering mechanism to allow them to use this technology without any wastes of resources. In MU-MIMO, the performance of the user bases on who is sending simultaneously. The existing MAC protocol of MU-MIMO enables all clients to use traditional MAC protocol and competes for the channel, by doing this, the medium time will be wasted and failed to deliver the earning of MU-MIMO.

Making clustering in uplink MU-MIMO is much more challenging than downlink mode because there is no coordination point. The best clustering mechanism that used with uplink MU-MIMO MAC protocol is proposed in [2], namely the MIMOMate matching mechanism. In MIMOMate, the chain or cluster of clients will be generated to enable them concurrent transmissions. This matching is based on grouping clients whose mediums are more orthogonal to each other to eliminate any interference and maximize the throughput. This MAC protocol that based on MIMOMate allows only at the first stream all clients to compete for the channel. After that, the residual clients connect the simultaneous transmission of its leader in the MIMO-Mate relation. As a result, the less contention environment will reduce the overhead. The proposed MU-MIMO MAC protocol based on MIMOMate [2] suffers from some limitations such as: it does not state clearly how the leader communicates with his members to start transmissions, and how the antennas communicate together. In addition, it uses an original backoff algorithm that influences the performance of the overall network. Based on the above reasons, the contribution of this paper is to implement the CMU-MIMO protocol that utilize MIMOMate clustering mechanism to deal with these limitations, maximize the throughput and re- 
duce delay. Figure 1 summarizes the main steps and the following four sub sections present detailed explanations of these steps.

\subsubsection{The Proposed CMU-MIMO Protocol}

To achieve the goal of our improvement, firstly, we assume a dense network. Secondly, the number of members in one cluster is identical to the number of antennas in the AP. Thirdly, each antenna (angle) has one member in each cluster and only the leader of the cluster will compete each time. Fourthly, each station has data to send and the transmission time of all stations relatively close to each other. Lastly, the nodes were clustered using the MIMOMate technique.

We propose the following CMU-MIMO algorithms as shown in Figure 2. The global variable for the antennas and local variable in each AP that we need to

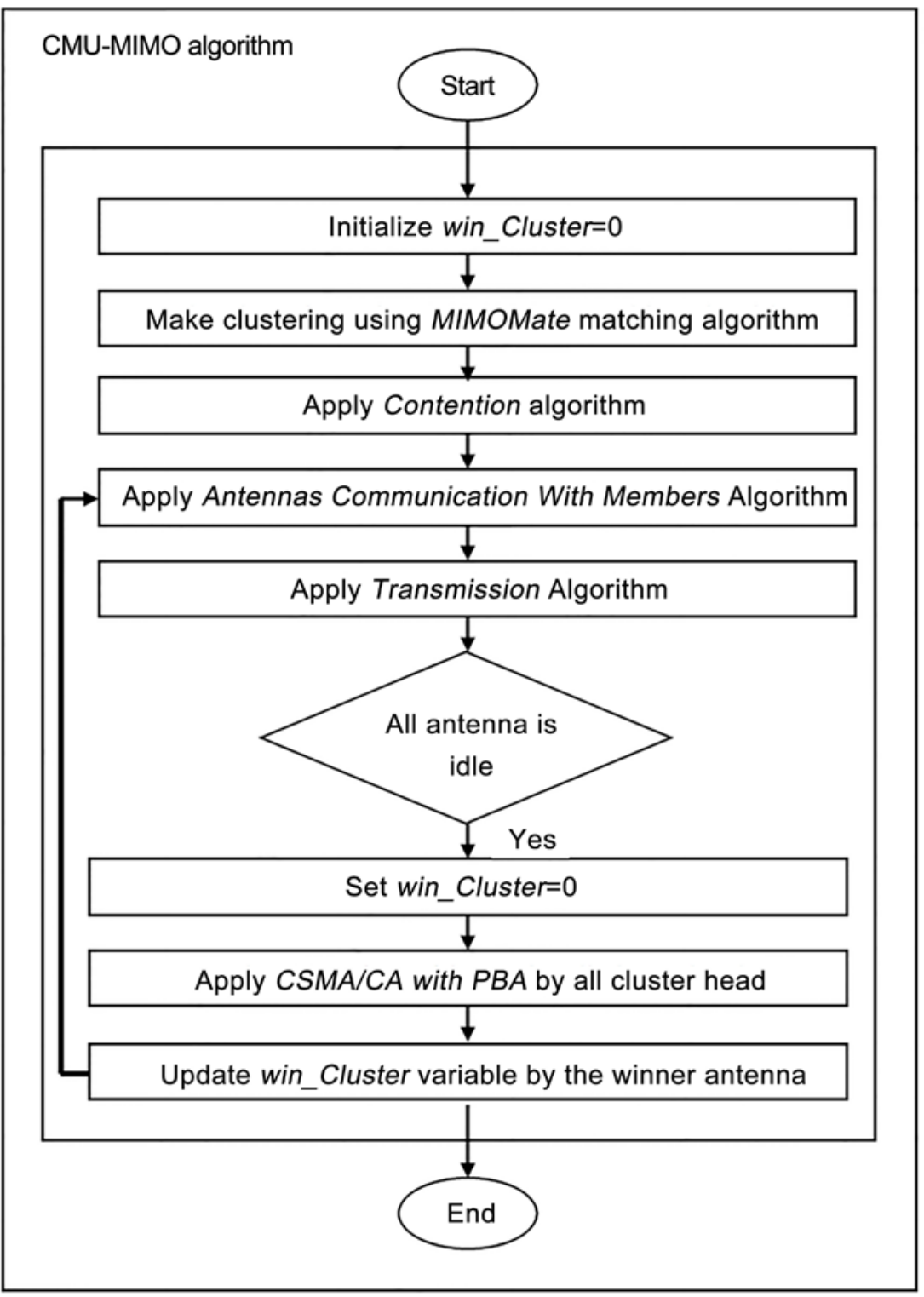

Figure 1. Flow diagram of CMU-MIMO. 
identify is the Win_cluster. It is used to store the number of the winning cluster in the contention process. After that, the variable used by all antennas tell the members of this cluster that they have to send in this time. The first step of the algorithm is to initialize the global variable as 0 , and then apply the MIMOMate matching algorithm to generate clusters. After that, calling the Contention Algorithm that allows the contention between leaders for the first stream to access the channel, which will be described in the following sub section.

\subsubsection{Contention Algorithm}

The main goal of this algorithm is to start the contention between all leaders when all antennas are idle after each cycle (one cycle means one cluster occupies the channel and has finish off sending). The steps of this algorithm are shown in Figure 3. First, set the variable Win-cluster to zero when new contention starts. Then apply CSMA/CA protocol that integrates PBA by all cluster heads, the antenna of the winner will update the value of Win-cluster. After that, all other antennas will know that the value of this variable is changed, and stored the number of new winner cluster. Then all another antenna will communicate with their stations by calling Antennas Communication with the Members Algori- thm. Finally, the cluster will start the transmission by calling the Transmission Algorithm. These algorithms are explained in the following two sub sections.

\subsubsection{Antennas Communication with Members Algorithm}

The main goal of this algorithm is to communicate each antenna with the members of the winning cluster. The steps of this algorithm are shown in Figure 4, the input of this algorithm is the number of the winner cluster. According to the number of antennas in the AP, this algorithm loops to check all antennas.

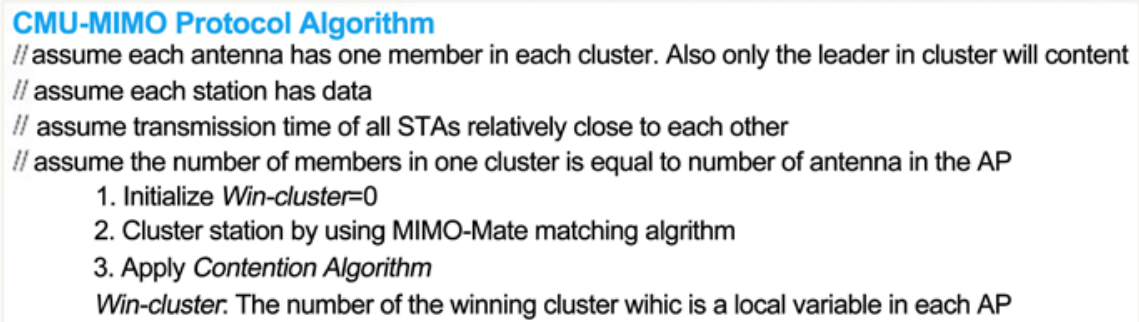

Figure 2. CMU-MIMO protocol algorithm.

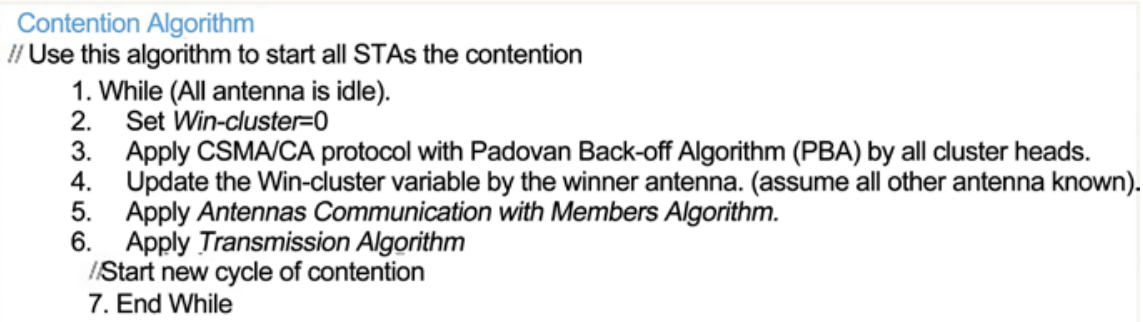

Figure 3. Contention algorithm that allow leaders to make contention when all antennas are idle after each cycle. 
If this antenna is the winner antenna, the algorithm will allow the antenna's station to start sending its data by using the mechanism of handshaking (RTS/ CTS). Otherwise, the antenna will check first if the number of the winner cluster matches its table to fetch the MAC address. After that, add it to the requestto-receive (RTR) frame header, and then broadcast this frame to all its stations. Therefore, all members of the winner cluster will know that they have to send their data in this cycle. In contrast, if there is no matching then it broadcast an error message.

\subsubsection{Transmission Algorithm}

This algorithm allows the member of the winner cluster to start its transmission concurrently as shown in Figure 5. At the beginning, the algorithm will first check if this antenna is not the winner antenna, and then check if the member (station) has data to send or not. If yes, then it starts sending RTS/CTS control message, at last, the station will send the data frames.

\section{Experimental Result and Analysis}

In this section, we present the implementation of CMU-MIMO protocol and test its performance. After that we discuss the improvement in performance of

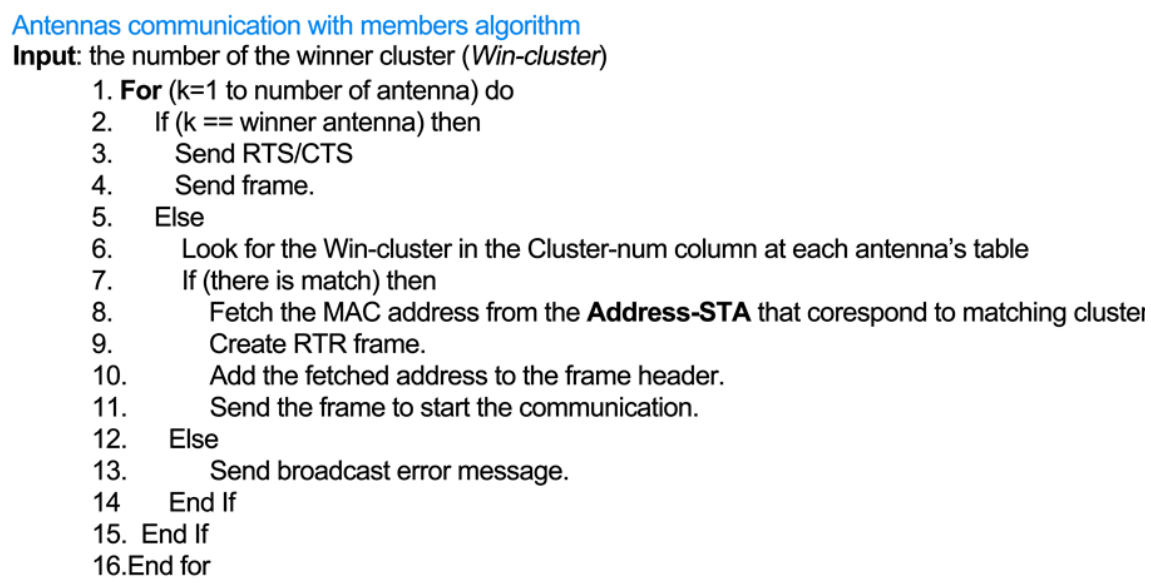

Figure 4. Antennas Communication with Members algorithm.

\section{Transmission Algorithm}

// the member of the winner cluster will send its data at the same time.

1. For ( $k=1$ (to number of antenna - 1)) do

2. if ( $k$ !=the winner antenna) then

3. If (STAs $k$ has data to send) then

4. Send RTS/CTS

$5 . \quad$ Send frame.

6. End If

7. End If

\section{End For}

Figure 5. Transmission algorithm which allows the member of the winner cluster to start its transmission concurentlly. 
CMU-MIMO protocol. We compared the performance of IEEE 802.11n protocol and the CMU-MIMO protocol in terms of throughput and average delay. For better analysis, we have tested our method on different network sizes. The following are the specifications of hardware and software, then the simulation setting we have used to conduct all experimental runs. After that, we present the implementation of PBA and its result. Finally, the implementation of CMUMIMO protocol and its result will describe.

\subsection{System Specification}

Determining the specification of a system is one of the factors that eases the understanding of results. In this section, we will specify the hardware and software requirement that we used to implement in this paper.

The hardware that used is a computer with $993.4 \mathrm{MB}$, two processors Intel(R) Core(TM)2 CPU and 4300 @1.80GHz processor speed. While the software is an Ubuntu operating system with 9.10 (karmic) release, Kernel Linux 2.6.31-14generic and GNOME 2.28.1. Moreover, we use NS-2.34 network simulator software. The programming languages that we used in our implementation are $\mathrm{C}++$ and OTCL.

\subsection{Simulation Setting}

To show the performance of implementing CMU-MIMO protocol, we specify the simulation setting parameters in our experiment as shown in Table 1. Also, we conduct the number of different scenarios that vary between 5 and 11 nodes (maximum size allowed based on memory available). After that, we show the behavior of CMU-MIMO protocol by measuring the throughput and average delay.

\subsection{Implementation of PBA and Result}

PBA is a new scheme that used as a way to reduce the collision that may happen in the IEEE 802.11n WLAN network. In this paper, we modify the 802.11n MAC protocol by replacing $\mathrm{BEB}$ with $\mathrm{PBA}$ and test its effectiveness on the performance of the network. Also, we compare this algorithm with the BEB as a benchmark. The implementation is carried on the system specification that mentioned above.

How the CW size update is the most affected parameter on the performance of network protocols. The PBA increases the $\mathrm{CW}$ of node up to the maximum value $\mathrm{CW}$ max after unsuccessful transmission, and resets it to the minimum value $\mathrm{CW}$ min after successful transmission according to the following Equation [9]:

$$
C W \text { new }= \begin{cases}\min \left(P^{*} C \text { Wold }, C W \max \right) & \text { if collision } \\ C W \max & \text { if success }\end{cases}
$$

Where P represents the Padovan number that calculated by this Equation:

$$
P(0)=P(1)=P(2)=1
$$


The recurrence relation is given by this Equation:

$$
P(n)=P(n-2)+P(n-3), n \leq 3
$$

How the CW increases comparing to the BEB CW is shown in Figure 6.

After applying BEB with PBA algorithms on different scenarios with different sizes of network, and taking the average of running these algorithms ten times, we conclude that as the network becomes dense, the throughput increases and the average delay decreases compared to the BEB.

Figure 7 shows the result obtained by plotting network size versus throughput that measured in kilo byte per second $(\mathrm{Kb} / \mathrm{s})$. As we can see that with the increasing of the number average throughput by $1.6 \%$ in $\mathrm{PBA}$ compared to BEB. This proves that the PBA has better and clear performance in large network size than a small one.

The next graph is obtained by plotting network size on the $\mathrm{x}$-axis and average delay on the $y$-axis that measured in milisecond ( $\mathrm{ms}$ ). As shown in Figure 8, the PBA has close and similar average delay to the BEB in small network size.

Table 1. Simulation setting parameter.

\begin{tabular}{cccc}
\hline Parameters & Value & Parameters & Value \\
\hline Access control & CSMA/CA & Number of node & 5-11 nodes \\
Antenna type & Direction Antenna & Packet size & 1500 bytes \\
Application protocol & FTP & Routing protocol & AODV \\
Bandwidth & 2Mbits/s & RTS Threshold & 3000 bytes \\
CW max & 1023 & DIFS & 10 us \\
CW min & 31 & Simulation time & $15 \mathrm{~ms}$ \\
MAC protocol & IEEE $802.11 \mathrm{n}$ & Slot time & 20us \\
Number of interfaces & 2 & Transport protocol & TCP \\
\hline
\end{tabular}

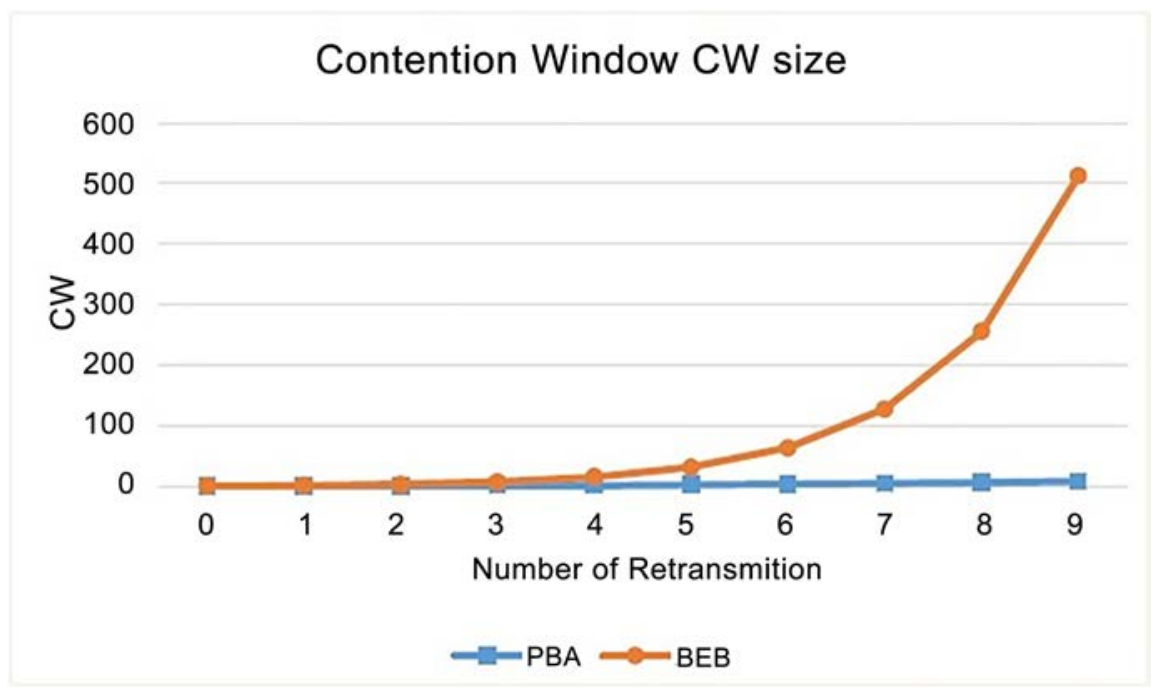

Figure 6. Contention window size. 


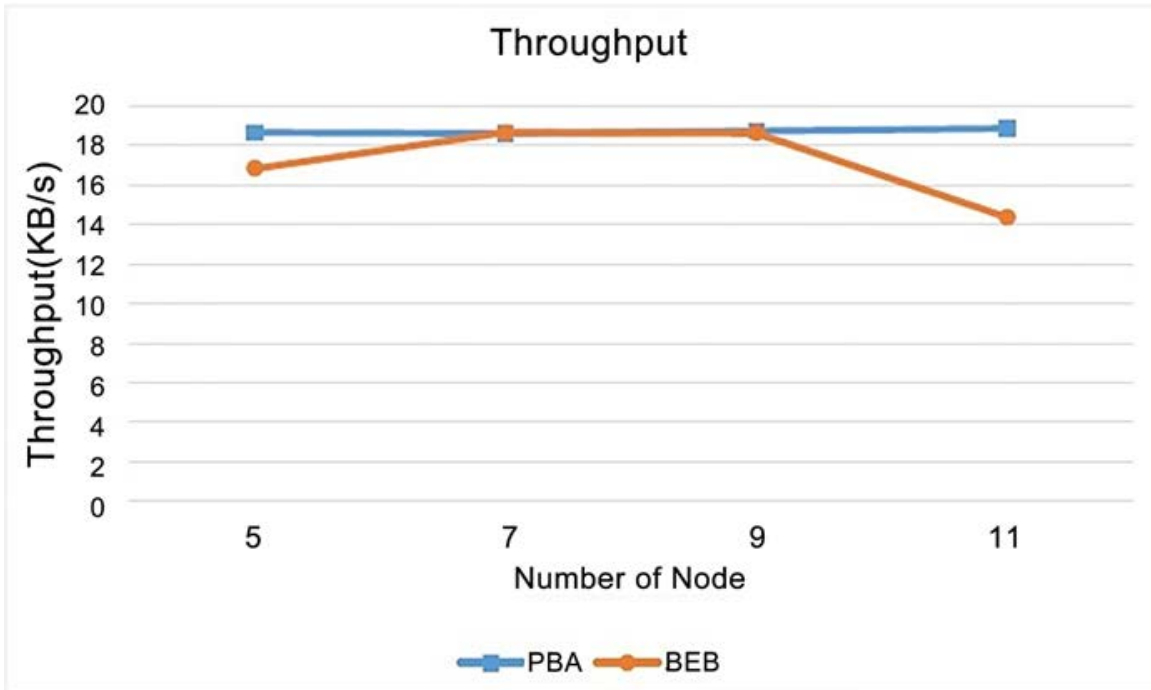

Figure 7. Throughput vs. Network size in CMU-MIMO protocol.

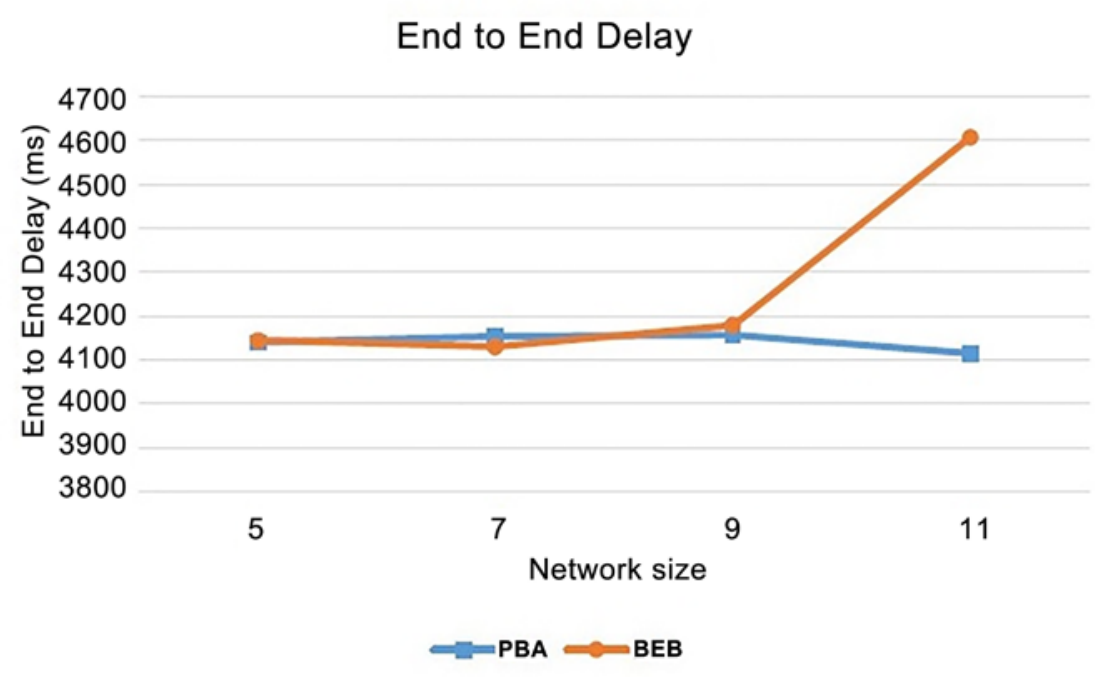

Figure 8. The end to end delay vs. network size in CMU-MIMO protocol.

However, when the number of nodes increases, PBA reduces the average delay compared to the BEB by $2.9 \%$. This result expected because the PBA increase its CW slowly and reduce the unnecessary time that nodes must wait to access the channel. From these results, we decide to use PBA instead of BEB in our proposed algorithm.

Finally, we have to mention that in both PBA and BEB the throughput and average delay are inversely proportional to the network size. This means that if the network is dense the number of contending nodes within the same transmission range increase that leads to lower throughput and average delay.

\subsection{Implementation of Clustering MU-MIMO Protocol and Result}

In our implementation, we apply many modifications on different files by adding the clustering concept. One of these files is mac_802.11n MAC protocol, 
which contains the main functions of handshaking mechanism. After that we compare CMU-MIMO protocol with the IEEE $802.11 \mathrm{n}$ protocol as a benchmark. In the experiments we executed the CMU-MIMO protocol and IEEE 802.11n protocol on four network sizes; firstly, with network size that allowed making a cluster in CMU-MIMO protocol which is five nodes. This is because we assume the number of nodes in each cluster is equal to the number of antennas that found in the AP (which is n0 in our experiment). Then increased to 7 nodes, nine nodes and lastly for 11 nodes (maximum size allowed based on memory available).

Moreover, we ran these two protocols in two cases, the first one is when there is no contention between leaders, while the other one is when there is a contention between leaders that locate and covered by the same antenna. After that, we ran both of these protocols ten times for each network size then we take the average of them in each case. The results obtained from the CMU-MIMO protocol are used to calculate the throughput and the average delay of each network size when we fixed the Transmission Control Protocol (TCP) connection between nodes. Figure 9 shows the scenario with 11 nodes and the channel is occupied by the members of first cluster $\mathrm{n} 1, \mathrm{n} 2$ and communicated with the AP (n0).

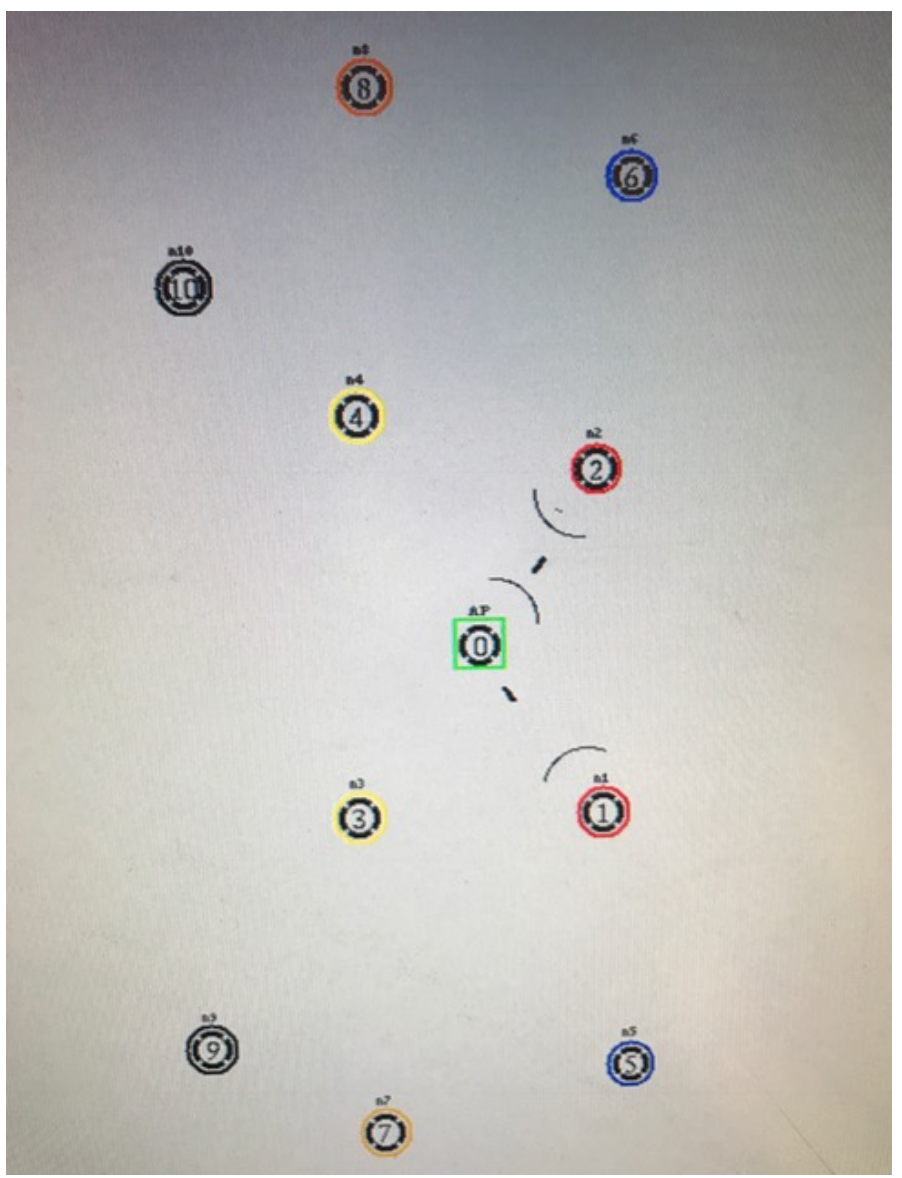

Figure 9. A sample of output scenario with 11 nodes, where $\mathrm{n} 0$ is the $\mathrm{AP}$ and the first cluster with red color (n1, n2) occupy the channel and communicate with the AP (n0). 
In this scenario, we have five clusters, in each one there are two nodes and n0 setting as AP. The description of this scenario is shown in Table 2. The next subsections discuss the results obtained from case 1 and case 2 AP.

\subsubsection{Scenario with No Contention}

As shown in Figure 10, we obtained the first case by plotting network size and throughput, which is measured in kilo byte per second $(\mathrm{Kb} / \mathrm{s})$, on $\mathrm{x}$ and $\mathrm{y}$-axis respectively. Each line represents the result of applying CMU-MIMO protocol and the IEEE 802.11n corresponding with different network sizes used in our experiments. As we can see that with the increasing of the number of nodes, there is a considerable increase in the throughput in CMU-MIMO protocol by $0.99 \%$, while in the IEEE $802.11 \mathrm{n}$, we can see that there is inconsistency in the value of through put.

The next graph is obtained by plotting network size on the $\mathrm{x}$-axis and average delay that, measured in millisecond (ms), on the y-axis. As shown in Figure 11, each line represents the result of applying CMU-MIMO protocol and the IEEE 802.11n corresponding with different network sizes used in our experiments. The graph shows clearly that CMU-MIMO minimizes the average delay by $0.06 \%$ in dense network.

Table 2. Scenario description.

\begin{tabular}{cccc}
\hline Parameters & Value & Parameters & Value \\
\hline 1 & Red & $\mathrm{n} 1$ and $\mathrm{n} 2$ & $\mathrm{n}$ 3 \\
2 & Yellow & $\mathrm{n} 3$ and $\mathrm{n} 4$ & $\mathrm{n} 5$ \\
4 & Blue & $\mathrm{n} 5$ and $\mathrm{n} 6$ & $\mathrm{n} 7$ \\
5 & Orange & $\mathrm{n} 7$ and $\mathrm{n} 8$ & $\mathrm{n} 9$
\end{tabular}

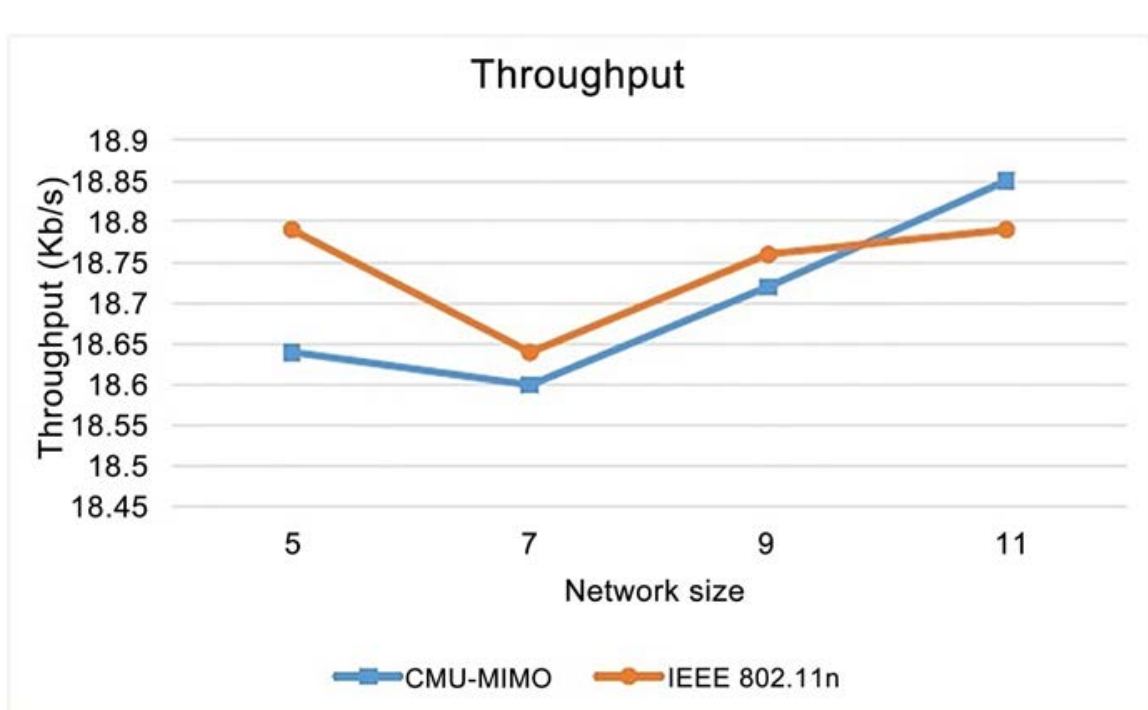

Figure 10. Throughput in the first case when there is no contention between nodes. 


\subsubsection{Scenario with Contention}

In the second case, as shown in Figure 12, we obtained by plotting network size and throughput, that measured in kilo byte per second $(\mathrm{Kb} / \mathrm{s})$, on $\mathrm{x}$ and $\mathrm{y}$-axis respectively. Each line represents the result of applying CMU-MIMO protocol and the IEEE 802.11n corresponding with different network sizes used in our experiments. As we can see clearly that CMU-MIMO protocol has better performance by $89.8 \%$ than IEEE $802.11 \mathrm{n}$ in term of throughput when there is a contention between nodes to access the medium. Therefore, we can conclude that the CMU-MIMO protocol solves the contention problem that IEEE $802.11 \mathrm{n}$ suffers from it, and lets all the nodes to have full and very high contention that leads to enter in a waiting time until the time is ended and each one waits for the other to start sending.

In Figure 13, we have plotted network size versus average delay that measured in millisecond (ms), where problem size is on the $\mathrm{x}$-axis and average delay is on

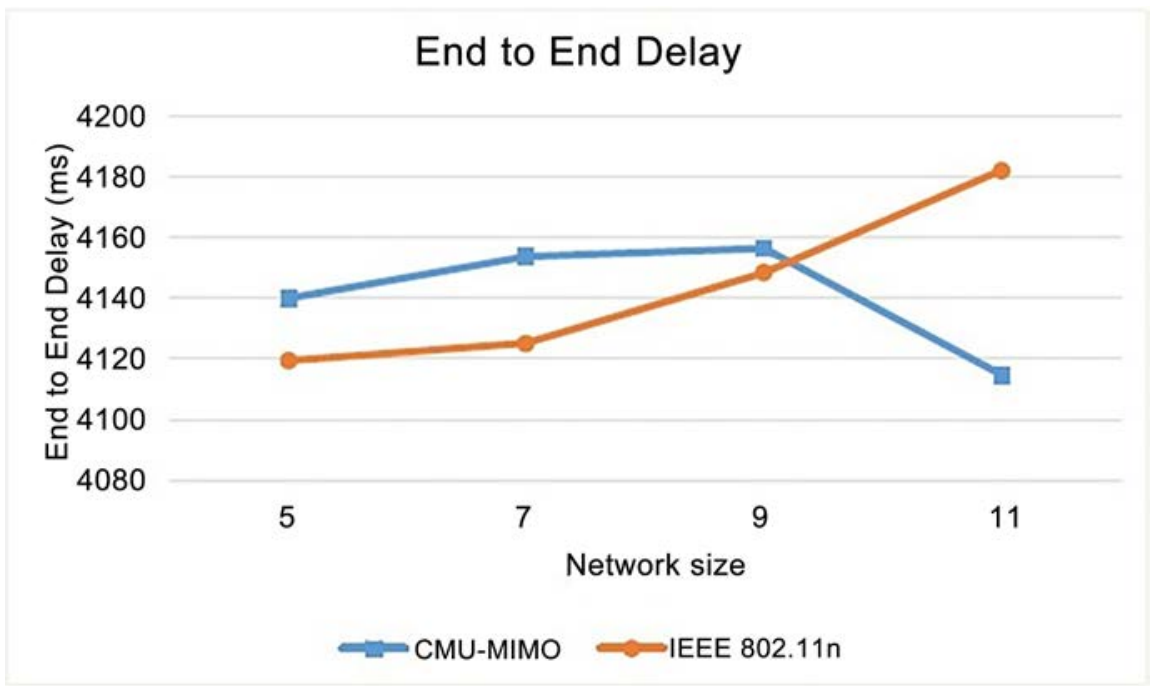

Figure 11. Average Delay in the first case where there is no contention between.

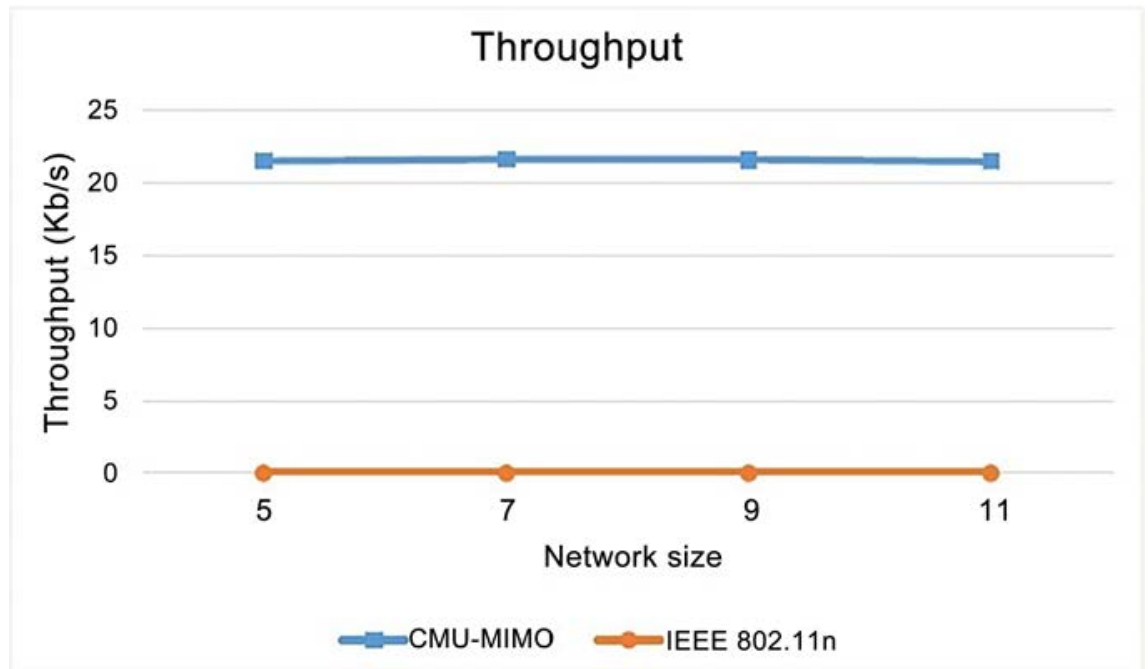

Figure 12. Throughput in the second case where there is a contention between. 


\section{End to End Delay}
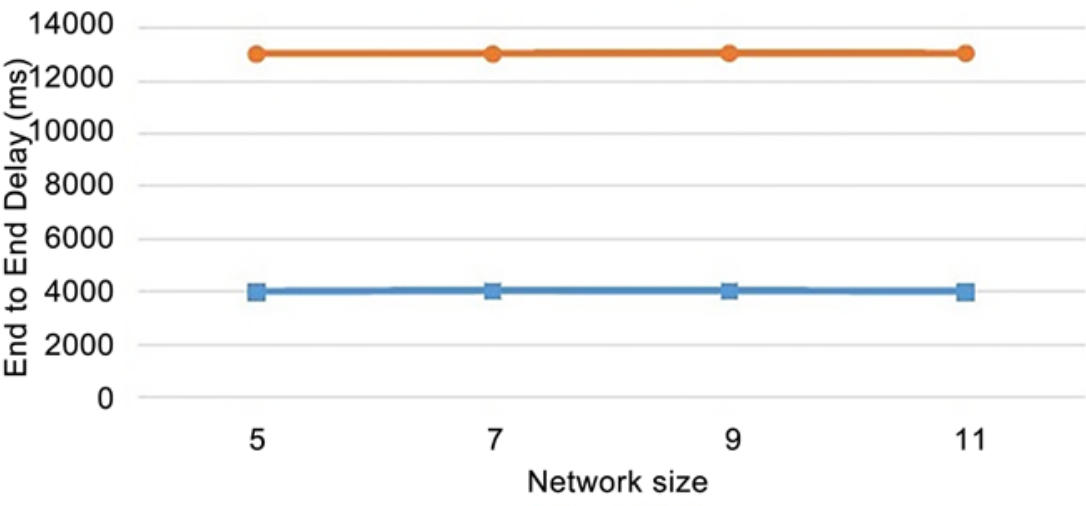

$=$ CMU-MIMO $\rightleftharpoons$ IEEE 802.11n

Figure 13. Average Delay in the second case where there is a contention between nodes.

the $y$-axis. As shown in this figure, each line represents the result of using a different number of nodes. It shows that CMU-MIMO protocol helps to decrease the average delay significant by $43.9 \%$.

From the results shown above, we can conclude that the CMU-MIMO protocol improves the performance of overall network by increasing the throughput and decreases the average delay.

\section{Conclusions and Future Work}

Recently, there is a noticeable increase in the number of wireless communication users. At the same time, the technologies that meet the requirements are improved. MU-MIMO is an advanced method of MIMO that can serve many users simultaneously, thus, the MU-MIMO improves the overall network communication. In this paper, we proposed a CMU-MIMO protocol, which is an improved MAC protocol for MU-MIMO based on MIMOMate mechanism and PBA. Based on our results, we concluded that CMU-MIMO protocol decreases the average delay by $43.9 \%$ and increases the throughput of wireless communication by $89.8 \%$. Therefore, a considerable improvement in performance of IEEE $802.11 \mathrm{n}$ is observed.

In future work, we will test CMU-MIMO in large network size by increasing the number of stations. Moreover, we will relax the assumptions in CMUMIMO protocol by increasing the numbers of members in one cluster, considering the case if the number of members is less than the number of antennas, utilizing all the antennas of AP in case of there are some stations in the winner cluster does not have data to send and if the transmission times between stations are different. Finally, measuring other metrics such as fairness to provide the quality of services will be considered.

\section{Acknowledgements}

The authors extend their appreciation to the Deanship of Scientific Research at 
King Saud University for funding this work through research group no. RGP264.

\section{References}

[1] Marzetta, T.L. (2015) Massive MIMO: An Introduction. Bell Labs Technical Journal, 20, 11-22. https://doi.org/10.15325/BLTJ.2015.2407793

[2] Kuo, T.-W., Lee, K.-C., Lin, K.C.-J. and Tsai, M.-J. (2014) Leader-Contention-Based User Matching for 802.11 Multiuser MIMO Networks. IEEE Transactions on Wireless Communication, 13, 4389-4400. https://doi.org/10.1109/TWC.2014.2320908

[3] Liao, R., Bellalta, B., Oliver, M. and Niu, Z. (2016) MU-MIMO MAC Protocols for Wireless Local Area Networks: A Survey. IEEE Communications Surveys \& Tutorials, 18, 162-183. https://doi.org/10.1109/COMST.2014.2377373

[4] Barghi, S., Jafarkhani, H. and Yousefi'zadeh, H. (2011) MIMO-Assisted MPR-Aware MAC Design for Asynchronous WLANs. IEEE/ ACM Transactions on Networking, 19, 1652-1665. https://doi.org/10.1109/TNET.2011.2130538

[5] Lin, K.C.-J., Gollakota, S. and Katabi, D. (2011) Random Access Heterogeneous MIMO Networks. Proceedings of the ACM SIGCOMM 2011 Conference, Toronto, 15-19 August 2011, 146-157. https://doi.org/10.1145/2018436.2018454

[6] Sadek, M., Tarighat, A. and Sayed, A.H. (2007) A Leakage-Based Precoding Scheme for Downlink Multi-User MIMO Channels. IEEE Transactions on Wireless Communications, 6, 1711-1721. https://doi.org/10.1109/TWC.2007.360373

[7] Jin, H., Jung, B.C. and Sung, D.K. (2011) A Tradeoff between Single-User and Multi-User MIMO Schemes in Multi-Rate Uplink WLANs. IEEE Transactions on Wireless Communications, 10, 3332-3342. https://doi.org/10.1109/TWC.2011.080311.101980

[8] Ettefagh, A., Kuhn, M., Eli, C. and Wittneben, A. (2011) Performance Analysis of Distributed Cluster-Based MAC Protocol for Multiuser MIMO Wireless Networks. EURASIP Journal on Wireless Communications and Networking, 2011, 34. https://doi.org/10.1186/1687-1499-2011-34

[9] Moad, D., Djahel, S. and Nait-Abdesselam, F. (2014) Padovan Sequence Based Backoff Algorithm for Improved Wireless Medium Access in MANETs. Global Information Infrastructure and Networking Symposium (GIIS), Montreal, 15-19 September 2014, 1-6. https://doi.org/10.1109/giis.2014.6934267

[10] Wang, K. and Sun, C. (2009) An Improved Backoff Algorithm of Ad Hoc Networks. International Conference on Computational Intelligence and Software Engineering (CiSE 2009), Wuhan, 11-13 December 2009, 1-4.

https://doi.org/10.1109/cise.2009.5362984

[11] Al-Hubaishi, M., Abdullah, T., Alsaqour, R. and Berqia, A. (2012) E-BEB Algorithm to Improve Quality of Service on Wireless Ad-Hoc Networks. Research Journal of Applied Sciences, Engineering and Technology, 4, 807-812.

[12] Li, T., Tang, T. and Chang, C. (2009) A New Backoff Algorithm for the IEEE 802.11 Distributed Coordination Function. 6th International Conference on Fuzzy Systems and Knowledge Discovery, Tianjin, 14-16 August 2009, 455-459.

https://doi.org/10.1109/fskd.2009.513 
Submit or recommend next manuscript to SCIRP and we will provide best service for you:

Accepting pre-submission inquiries through Email, Facebook, LinkedIn, Twitter, etc. A wide selection of journals (inclusive of 9 subjects, more than 200 journals)

Providing 24-hour high-quality service

User-friendly online submission system

Fair and swift peer-review system

Efficient typesetting and proofreading procedure

Display of the result of downloads and visits, as well as the number of cited articles Maximum dissemination of your research work

Submit your manuscript at: http://papersubmission.scirp.org/

Or contact cn@scirp.org 